Reprod. Nutr. Dévelop., 1988, 28 (1), 1-17.

\title{
Les orientations du métabolisme intermédiaire chez les ruminants
}

\author{
C. DEMIGNÉ, C. YACOUB, C. MORAND, C. RÉMÉSY
}

Laboratoire des Maladies Métaboliques I.N.R.A., Theix, 63122 Ceyrat, France.

Summary. Factors involved in metabolic adjustments in ruminants.

In ruminants, the digestive tract and liver metabolism represents a substantial part of the energy requirements. There is almost no net glucose absorption by the gut although it is an energy fuel for the enterocytes. There is a permanent gluconeogenesis, for which the major substrate is propionate. Propioniate plays a major role in glucose synthesis is highly effective and propionate metabolism may affect the utilization of other substrates such as lactate. In addition, amino acid metabolism provides a net supply of carbon for gluconeogenesis. However, there is a maximal sparing of the $\mathrm{NH}_{2}$ group (via glutamate release) by the ruminant liver and the energy cost of the conversion of amino acids into glucose is very high.

The actual importance of insuline in the regulation of liver metabolism in the ruminant is not entirely understood but glucagon seems not to be a major factor in the stimulation of gluconeogenesis in the fed ruminant.

Propionate has a potent effect on anabolism because of its effects on glycaemia and insuline secretion. When glucose requirements are to be enhanced (pregnancy, lactation), even a minor imbalance in the supply of glucogenic substrates may elicit striking physiological changes such as lipomobilization or ketonaemia.

In early lactation, insuline secretion is still small in spite of an enhanced food intake. As a result, glucose, fatty acids and amino acids are increasingly chanelled towards udder metabolism. Lipogenesis is depressed whereas lipolysis is stimulated by catecholamines. However, excessive glucose and propionate supply may lead to exagerated anabolism and to a drop in milk fat. Thus, a high milk production requires an optimal supply of the three volatile fatty acids (VFA) and it should be emphasized that an improved nitrogen supply has a stimulatory effect on milk production.

Les besoins des ruminants peuvent être couverts par des régimes comprenant des proportions très diverses de fourrages, de céréales, de parois cellulaires et d'amidon. Il en résulte une répartition très différente des substrats énergétiques absorbés pour les synthèses corporelles, les besoins du fœtus ou la sécrétion lactée. Les orientations métaboliques chez les ruminants sont donc particulièrement complexes puisqu'elles résultent $d$ 'une disponibilité très diverse en nutriments pour des états endocriniens très variables. Nous traiterons dans cet article principalement de l'orientation du métabolisme intermédiaire en mettant l'accent sur le 
rôle du tube digestif et du foie. L'autre partie sera consacrée aux orientations du métabolisme protéique et de sa régulation par les hormones (Grizard et al., 1988).

\section{Importance du métabolisme digestif et hépatique.}

Le foie et la muqueuse gastrointestinale sont parmi les tissus les plus actifs d'un point de vue métabolique (Huntington et Reynolds, 1987). Ils consommeraient près de $1 / 3$ de l'énergie ingérée par les vaches en lactation. Smith et Baldwin (1974) estiment que l'augmentation de taille du tube digestif et du foie représentent respectivement 25 et $56 \%$ de l'élévation des besoins d'entretien de vaches en lactation. Par rapport à des animaux recevant du foin, le poids du foie est $20 \%$ plus élevé chez les ruminants nourris avec du concentré (Martin et al., 1973).

La surface d'échange des papilles du rumen peut augmenter de 3 à 5 fois, en fonction de la quantité d'énergie ingérée, et l'absorption des acides gras volatils augmente près de 3 fois pendant les 4-6 premières semaines de lactation chez des vaches de production élevée (Dirksen et al., 1984). Le maximum de poids de l'épithélium ruminal et du foie est observé à la $6^{\mathbf{e}}$ semaine de lactation (Fell et al., 1972). Selon Bauman et Currie (1980), la prolactine serait impliquée dans I'hypertrophie et l'augmentation des capacités absorptives du tube digestif de la rate en début de lactation.

\section{Métabolisme spécifique de l'épithélium du tube digestif.}

\section{Rumen.}

Il existe une utilisation importante de glucose par l'épithélium du rumen, avec production d'acide lactique, qui provient en partie de la glycolyse dans les couches musculaires. Le glucose utilisé est exclusivement d'origine artérielle. Parallèlement, des quantités notables de glutamine artérielle sont captées : ceci est une caractéristique des tissus à renouvellement rapide et est à attribuer au métabolisme de la muqueuse.

Un certain potentiel de métabolisation du propionate, avec production de lactate, est présent dans l'épithélium du rumen; en fait, l'activation du propionate en propionyl-CoA est très fortement inhibée en présence de concentrations physiologiques de butyrate (Elliott, 1980).

II existe une cétogenèse ruminale importante qui se traduit par une libération de 3-hydroxybutyrate (3-HOB). La majorité du 3-HOB produit dans l'épithélium ruminal provient du butyrate, avec une très faible contribution de l'acétate et des acides gras libres (AGL) à l'état nourri (Leng et West, 1969). L'élévation du 3HOB plasmatique que l'on observe en début de lactation chez la vache (Stangassinger et Giesecke, 1984) peut être de même reliée à la croissance de l'épithélium ruminal faisant suite à la régression qui a eu lieu pendant la période de tarissement. 
La proportion de butyrate transformée en $\mathrm{CO}_{2}$ et corps cétoniques dépend de la concentration en butyrate (Beck et al., 1984). La conversion d'une large part du butyrate en 3-HOB par la muqueuse ruminale permet à ces chaînons à $4 \mathrm{C}$ de franchir la barrière hépatique, faisant ainsi parvenir un substrat énergétique directement aux tissus périphériques. De plus, le butyrate, qui est un régulateur des divisions cellulaires y compris au niveau de l'épithélium du rumen (Sakata et Yajima, 1984), est ainsi diminué.

\section{Intestin grêle.}

La majeure partie des substrats digestibles ingérés par les ruminants est dégradée par la flore ruminale et, dans de nombreux cas, il n'existe pas d'absorption notable de glucose par le tube digestif. Du fait que certains types de cellules de l'intestin (en particulier les entérocytes) opèrent une glycolyse importante, il existe généralement une consommation nette de glucose par le tube digestif. Toutefois, il est établi que certaines rations riches en concentrés, surtout lorsqu'elles sont à base de maïs, permettent l'arrivée de quantités notables d'amidon dans l'intestin grêle et il existe une absorption nette de glucose à ce niveau (Huntington et al., 1981) malgré la production de lactate par les entérocytes.

L'épithélium de l'intestin grêle est l'un des principaux sites d'utilisation de la glutamine dans l'organisme ; cependant une large part est recyclée en alanine, comme chez les monogastriques, ainsi qu'en acides aminés du cycle de l'urée, notamment en citrulline (Heitmann et Bergman, 1980).

L'intestin grêle peut tirer une partie de son énergie de l'utilisation des corps cétoniques dont une large part peut provenir de la production de 3-HOB par la paroi du rumen. Les bilans portaux en glucose, lactate, et corps cétoniques résultent donc de flux métaboliques digestifs complexes. Le fait que le tube digestif ait un besoin élevé en glucose et en acides aminés, en particulier au cours de son développement (début de lactation), pourra rendre encore plus critique I'homéostasie glucidique.

\section{Le rôle du foie dans le métabolisme intermédiaire.}

Alors que la cétogenèse est principalement ruminale chez l'animal nourri la néoglucogenèse est surtout hépatique; cependant, les reins pourraient rendre compte de $15 \%$ du glucose synthétisé (Bergman et al., 1974).

Chez le monogastrique, le foie joue un rôle important dans l'utilisation du glucose et contribue à l'homéostasie glucidique en captant une partie du glucose absorbé dans le tube digestif. Les possibilités d'utilisation tu glucose par le foie des ruminants semblent excessivement faibles (absence de glucokinase). Le glucose exogène ne devrait donc pas influencer directement le métabolisme hépatique et inhiber par exemple la néoglucogenèse, comme chez le monogastrique. Pourtant le glucose peut avoir une action régulatrice sur le métabolisme du glycogène chez le ruminant (Baird et al., 1980 ; Chaiyabutr et al., 1983).

Les effets du glucose exogène consisteraient à favoriser la synthèse de glycogène à partir des précurseurs glucogéniques (Katz et McGarry, 1984) aux dépens 
de la libération du glucose, mais sans variation du glux de néoglucogenèse (NG). La vitesse de la NG est maximale lorsque la disponibilité des précurseurs glucoformateurs est très élevée (Bergman et al., 1970) et il ne semble pas exister de limitation, même lorsque des quantités notables de glucose sont absorbées dans la veine porte avec certains régimes très riches en concentrés (Wieghart et al., 1986).

\section{TABLEAU 1}

Les différents effets du propionate, ou de ses dérivés, sur le métabolisme hépatique.

Effet $\quad$ Mécanisme

Propionate

\section{Dérivés Acyl-CoA}

Propionyl-CoA

Méthylmalonyl-CoA

Propionyl-CoA

Succinyl-CoA

Propionyl-CoA $(+)$

Méthylmalonyl-CoA (-)

Succinyl-CoA (?)
Inhibition de l'uréogenèse?

Inhibition de la cétogenèse

\section{Compétition pour l'activation en acyl-CoA avec les autres acides gras volatils}

Favorise l'entrée dans les mito chondries des charges + (ex. : $\mathrm{NH}_{4}+$ )

Inhibition de l'entrée des acyl-CoA dans la mitochondrie

Inhibition de la $\beta$-oxydation

Modulation de la néoglucogenèse à partir du pyruvate
Diminue le $[$ CoAl libre

Effet anion perméant
Compétition avec $\mathrm{Ac}-\mathrm{CoA}$ pour la synthèse de $\mathrm{N}$-acétylglutamate*

Simule l'effet du maionyl-CoA sur l'ACT I**

" suicide-inactivation 》 d'une FAD-déshydrogénase***

succinylation de I'HMG-CoA synthétase $e^{* * * *}$

Interactions avec le site allostérique de l'acétyl-CoA

\section{Acides organiques dérivés du propionate}

Méthylmalonate

Oxaloacétate

Oxaloacétate
Inhibition du transport du malate hors des mitos.

Inhibition de la cétogenèse

Activation de l'uréogenèse compétition ?

Détournement de l'acétyl-CoA vers le cycle de Krebs

Favorise la sortie hors de la mitochondrie de $-\mathrm{NH}_{2}$ sous forme d'aspartate.

* Les effets sur l'uréogenèse sont complexes, puisqu'ils sont la résultante d'effets activateurs ou inhibiteurs : ainsi, globalement, le propionate semble plutôt activateur chez le ruminant (Demigné et al., 1986) alors qu'il est inhibiteur chez le rat (Coudé et al., 1979). ** Brindle et al., 1985; *** Shaw et Engel, $1985 ; * * * *$ Lowe et Tubbs, 1985.

ACT : acylcarnitine transférase ; HMG : hydroxyméthylglutaryl. 


\section{Le rôle déterminant de la disponibilité en propionate.}

Des travaux récents du laboratoire, sur hépatocytes isolés, ont permis de préciser l'efficacité relative des différents substrats glucoformateurs chez le ruminant et le rat (Demigné et al., 1986). Le précurseur le plus important chez le ruminant est indiscutablement le propionate. II pourrait fournir plus de $50 \%$ du glucose produit chez l'animal nourri (Bergman et al., 1970 ; Judson et Leng, 1973 ; Baird et al., 1980) et son utilisation pour la synthèse du glucose est directement proportionnelle à sa concentration dans le plasma portal. De nombreux travaux se sont efforcés d'établir la contribution respective des différents précurseurs à la production hépatique du glucose. Beaucoup citent des taux de conversion du propionate en glucose relativement faibles (parfois inférieurs à $50 \%$ ) ce qui, compte tenu de la faible captation hépatique du lactate à l'état nourri, oblige à attribuer par défaut une contribution exagérée aux acides aminés, voire au glycérol. Or, des données plus anciennes (Wiltrout et Satter, 1972) ont montré que le métabolisme du propionate dans le cycle de Krebs se limite à la séquence menant du succinylCoA à l'oxaloacétate, puis vers la synthèse de phosphoénolpyruvate. De même, les données in vitro, indiquent un taux de conversion du $2\left[{ }^{14} \mathrm{C}\right.$-propionate en glucose de l'ordre de $70 \%$ (Demigné et al., 1986). L'importance du propionate dans la synthèse hépatique du glucose chez le ruminant doit être réévaluée et est généralement bien supérieure à $50 \%$, d'autant plus que le propionate tend à diminuer l'utilisation des autres substrats glucoformateurs. Avec des régimes particulièrement favorables aux fermentations propioniques la majeure partie de glucose pourrait alors provenir de l'apport de propionate, en particulier si certaines enzymes clés de son métabolisme sont inductibles dans ces conditions (Wahle et al., 1981). L'élévation de la disponibilité en propionate réduit l'utilisation des autres substrats glucoformateurs, en particulier du lactate et du pyruvate (fig. 1)

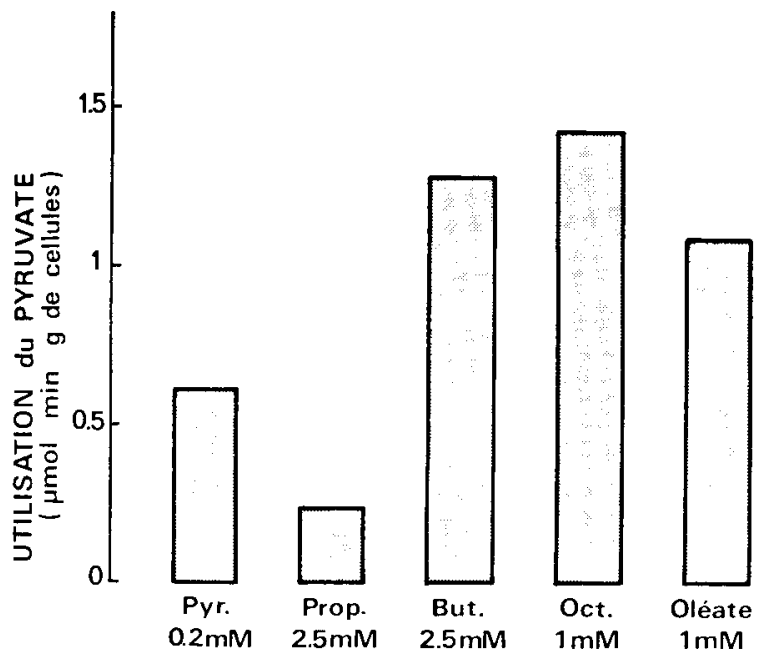

FIG. 1. -- Effets des divers acides gras sur l'utilisation du pyruvate $2 \mathrm{mM}$ par les hépatocytes isolés de mouton (Pyr. = pyruvate, Prop. = propionate, But. = butyrate, Oct. = octanoate). 
(Baird et al., 1980). Ceci s'explique par le fait que le propionate entre dans la voie de la NG au-delà de l'étape de la pyruvate carboxylase, à la différence du pyruvate et du lactate. Les possibilités d'inhibition de l'utilisation des autres substrats glucoformateurs par le propionate sont encore mal connues. L'utilisation du propionate pour la synthèse du glucose ne semble pas nettement influencée par les hormones.

In vitro, par rapport à un monogastrique tel que le rat, il est clair que les hépatocytes de ruminant utilisent difficilement le L-lactate, le glycérol, l'alanine ainsi que le pyruvate. Ces études confirment que le ruminant possède des capacités d'utilisation du propionate exceptionnellement élevées (Demigné et al., 1986). Il est remarquable que ni le glucose à forte concentration (10 $\mathrm{mM})$, ni l'oléate (source d'énergie) n'affectent la NG à partir du propionate (tabl. 2). Hormis le cas du butyrate (compétition éventuelle pour l'activation; Ricks et Cook, 1978) seul $\mathrm{NH}_{3}$ diminue la conversion du propionate en glucose, sans doute au profit de la synthèse de certains acides aminés (Demigné et al., 1986). Ces données corroborent celles obtenues in vivo : prédominance du propionate, faible néoglucogenèse à partir du lactate (Naylor et al., 1984), ce qui peut sembler paradoxal pour une situation de NG active.

TABLEAU 2

Effets de divers substrats sur la vitesse d'utilisation du propionate et de sa conversion en glucose (d'après Demigné et al., 1986).

\begin{tabular}{lcc}
\hline Addition & $\begin{array}{c}\text { Néoglucogenèse } \\
\text { à partir du propionate }\end{array}$ & $\begin{array}{c}\text { Utilisation } \\
\text { du propionate }\end{array}$ \\
\hline Aucune & $0,56 \pm 0,05$ & $1,56 \pm 0,12$ \\
Butyrate $(2 \mathrm{mM})$ & $0,21 \pm 0,03^{*}$ & $0,66 \pm 0,05^{*}$ \\
Ammoniac $(2 \mathrm{mM})$ & $0,35 \pm 0,04^{*}$ & $1,25 \pm 0,10^{*}$ \\
Oléate $(1 \mathrm{mM})$ & $0,59 \pm 0,06$ & $1,61 \pm 0,14$ \\
Glucose $(10 \mathrm{mM})$ & $0,55 \pm 0,05$ & $1,49 \pm 0,09$ \\
\hline
\end{tabular}

Les hépatocytes de mouton ont été incubés en présence de $2 \mathrm{mM}$ de $\left[2-{ }^{14} \mathrm{C}\right]$ propionate pendant $30 \mathrm{~min}$ à $37^{\circ} \mathrm{C}$. Les résuitats présentés sont la moyenne ( \pm E.S.M.) pour des mesures en double sur 5 lots différents de cellules et sont exprimés en $\mu$ moles $/ \mathrm{min}$ par $\mathrm{g}$ de cellule (poids frais).

* Différence significative par rapport aux conditions d'incubations avec le propionate seul.

Par contre, l'utilisation du lactate peut s'élever en cours de lactation ou de gestation (Bauman et Currie, 1980 ; Van der Walt et al., 1983). En principe, le Llactate ne représente pas un apport net de constituant glucoformateur à l'organisme, puisqu'il provient essentiellement du recyclage du glucose circulant (muscles lisses, intestin, placenta...) ou de la glucogénolyse périphérique. Le ruminant dispose de possibilités d'apport digestif net de lactate, essentiellement par glycolyse intestinale, alors que les autres processus (conversion de propionate en lactate par la paroi du rumen, absorption de L et D-lactate d'origine fermentaire) sont certainement mineurs. En fait, hormis les cas d'indigestion lactique, l'absorption digestive du lactate est certainement très limitée. 
Couplage de la néoglucogenèse et de l'uréogenèse (fig. 2).

Les acides aminés constituent par contre un apport net de chaînons glucoformateurs, bien qu'il s'y superpose aussi des phénomènes de recyclage entre tissus splanchniques et périphériques. Les principaux acides aminés glucoformateurs captés sont l'alanine, la glutamine, la glycine et dans une moindre mesure la sérine et la valine (Heitman et Bergman, 1980 ; Lindsay, 1982). La forte utilisation de la glutamine à l'état nourri est très spécifique du ruminant (elle est pratiquement nulle chez le monogastrique nourri, Rémésy et al., 1978) et se retrouve sur hépatocytes isolés. Par ailleurs, la glutaminase chez le ruminant se distingue par sa faible sensibilité à $\mathrm{NH}_{3}$ (puissant activateur chez les monogastriques) alors que l'inhibition par l'acidose reste présente (Yacoub et al., 1988). Cependant, le bilan hépatique en acides aminés est contrebalancé par une libération considérable de glutamate. D'autre part, comme le souligne Lindsay (1982), le taux de conversion des acides aminés en glucose peut être très inférieur à $100 \%$, notamment en ce qui concerne la glycine et la sérine, voire la glutamine. L'uréogenèse à partir des acides aminés implique une consommation d'énergie importante ; il faut 10 ATP pour fabriquer une molécule de glucose et d'urée à partir de

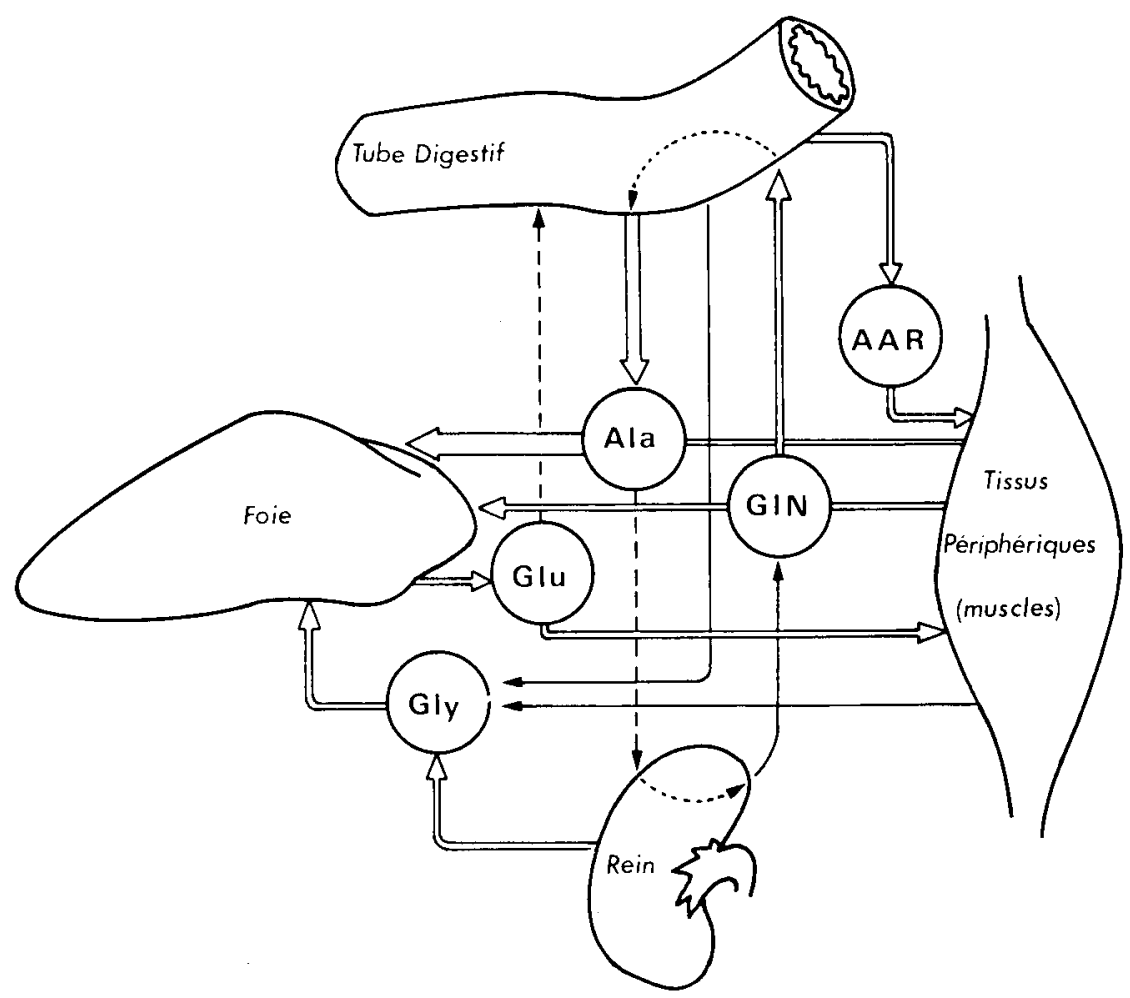

FIG. 2. - Relations interorganes impliquées dans le métabolisme des principaux acides aminés chez le mouton (Ala = alanine, Gly $=$ glycine, Glu = glutamate, GIN = glutamine, AAR = acides aminés ramifiés). 
2 molécules d'alanine, alors que l'oxydation complète du glucose fournit 38 ATP. Dans ces conditions, l'énergie peut provenir des acides gras longs mais il a aussi été montré in vitro que les acides gras volatils sont très efficaces à cet égard, en particulier le butyrate et dans une moindre mesure le propionate.

A jeun, et dans les situations de déficit énergétique où l'apport de propionate est très limitant, la contribution du lactate et des acides aminés à la synthèse du glucose augmente de façon importante ; de plus, le glycérol libéré au cours de la lipolyse dans le tissu adipeux tend à jouer un rôle notable.

\section{Le contrôle de la cétogenèse hépatique.}

Chez les ruminants la cétogenèse hépatique n'est importante que dans une situation de jeûne ou dans les cas d'acétonémie (fin de gestation, début de lactation). C'est pourquoi Heitmann et al. (1987) ont émis l'hypothèse que le 3-HOB d'origine digestive pouvait directement inhiber la cétogenèse hépatique. II agit certainement de concert avec le glucose et l'insuline pour diminuer la lipolyse. La cétogenèse hépatique peut être inhibée par le malonyl-CoA ou d'autres esters de CoA (méthylmalonyl-CoA, acétyl-CoA). Tous ces esters ont la propriété d'inhiber l'entrée des acyl-CoA dans la mitochondrie en agissant sur l'acyl-carnitine transférase 1. En plus de ce contrôle, les composés glucoformateurs peuvent améli-rer l'utilisation de l'acétyl-CoA dans le cycle de Krebs.

\section{Modulation du métabolisme intermédiaire par les hormones.}

Bien que la disponibilité en substrats soit déterminante pour contrôler la néoglucogenèse et la cétogenèse, les hormones exercent un effet important pour tempérer les changements brusques de l'apport de composés glucoformateurs et permettent aussi de répondre aux variations de la demande par les divers tissus.

En cas de déficit énergétique sévère, le manque de substrats rend indispensable une stimulation de la néoglucogenèse (mobilisation des substrats endogènes, meilleure conversion de ces substrats en glucose) par un nouvel état endocrinien caractérisé en particulier par une baisse du rapport insuline/glucagon.

\section{Insuline.}

Pendant longtemps, le ruminant a été considéré comme moins sensible à l'insuline que le monogastrique. Ceci peut refléter le fait que la néoglucogenèse (en particulier à partir du propionate) est moins adaptable que chez le monogastrique. Par ailleurs, l'utilisation du glucose semble être moins sensible à l'insuline chez le ruminant, ce qui reflète peut être le fait que, en raison de l'importance de l'acétate, l'utilisation du glucose s'effectue surtout dans les tissus où elle est peu adaptable (système nerveux et cellules sanguines). L'inhibition de la libération hépatique du glucose par l'insuline est due en partie à la stimulation de la synthèse du glycogène mais cette hormone peut aussi inhiber la néoglucogenèse (Brockman, 1985) sans doute de façon indirecte. A des taux moyens ou élevés en insuline, la captation hépatique du pyruvate, de l'alanine et de la glutamine est aussi réduite. 
L'insuline exercerait aussi un effet anticétogène par la stimulation de l'acétylCoA carboxylase (inhibition de l'acylcarnitine transférase par le malonyl-CoA). Cette stimulation de la lipogenèse hépatique ne représente pas un phénomène quantitatif important mais joue par contre un rôle déterminant dans l'orientation du métabolisme hépatique. Dans ces conditions une partie des substrats glucoformateurs pourrait être utilisée pour la lipogenèse mais it n'est pas facile de mettre en évidence une diminution de leur efficacité néoglucogénique.

\section{Glucagon.}

De même que chez les autres espèces, le foie des ruminants possède des récepteurs à l'insuline et au glucagon dont la sensibilité varie en fonction des situations nutritionnelles et physiologiques (Grizard et al., 1986). Le glucagon est une hormone hyperglycémiante chez le ruminant ; toutefois il ne semble pas jouer
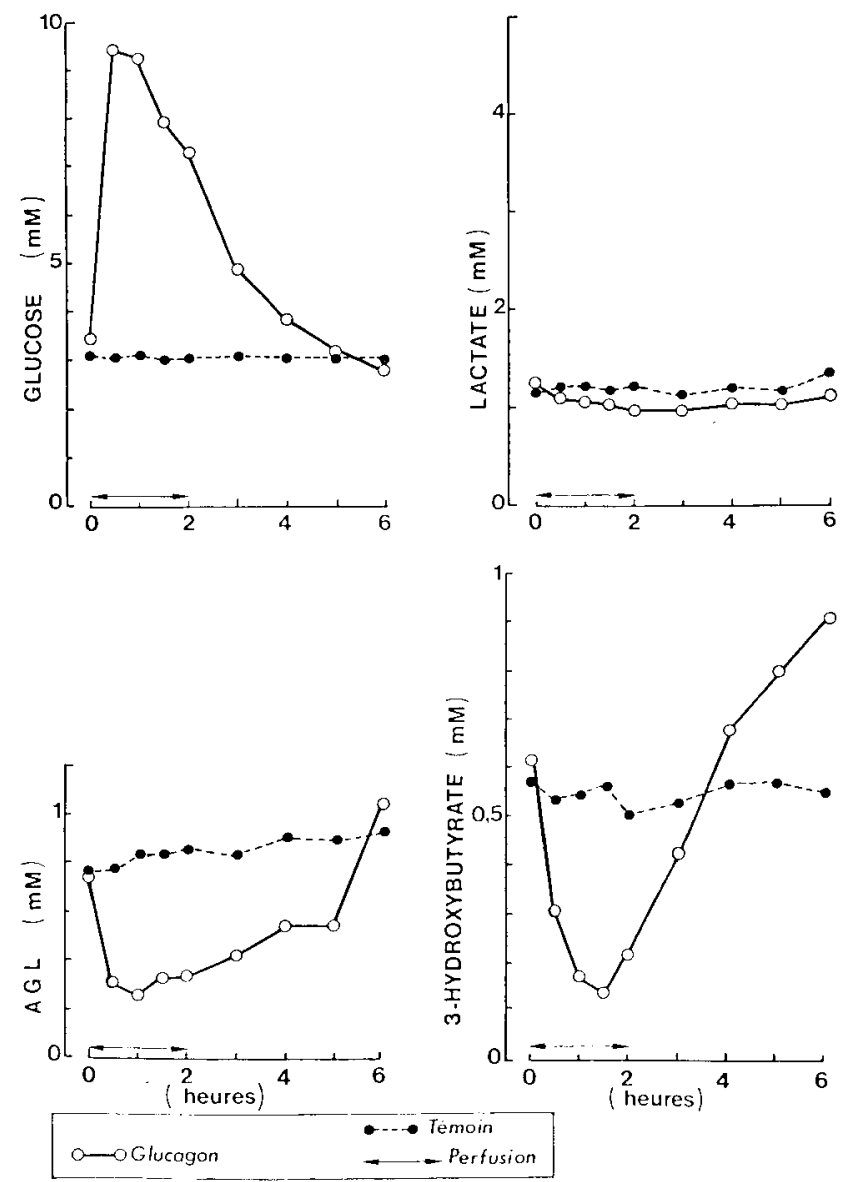

FIG. 3. - Effets d'une perfusion de glucagon à raison de $55 \mathrm{ng} / \mathrm{kg} / \mathrm{min}$ (après une première dose de $0,1 \mathrm{mg}$ ) à des brebis vides à jeun depuis $48 \mathrm{~h}$. 
un rôle déterminant dans l'augmentation de la NG à l'état nourri. In vivo la perfusion du glucagon n'a aucun effet lipolytique immédiat à la différence des catécholamines. Son effet hyperlipémiant stimule la sécrétion d'insuline, ce qui peut provoquer une baisse de la lipolyse et de la cétogenèse (fig. 3 ).

Chez le monogastrique, il est établi que le glucagon exerce ses effets via I'AMPc et le calcium (Mauger et Claret, 1986). Le glucagon affecte les diverses étapes clés de la synthèse du glucose en inhibant les enzymes de la glycolyse (phosphofructokinase et pyruvate kinase) et en stimulant les principales enzymes néoglucogéniques, notamment la phosphoénol pyruvate carboxykinase. Parallèlement, le glucagon a de nombreux effets sur le métabolisme mitochondrial qui se traduisent par une production plus importante d'ATP et d'acétyl-CoA, une accélération de certaines étapes du cycle de l'urée. Les effets néoglucogéniques du glucagon pourraient aussi être dus à une augmentation de la pyruvate carboxylase (Brockman et Laarveld, 1986). Nous avons vu que la pyruvate carboxylase étant sans doute l'étape limitante de I'utilisation du lactate; le glucagon pourrait donc agir à ce niveau pour stimuler l'utilisation du lactate. Toutefois, ceci n'est pas observé à partir d'hépatocytes isolés, alors même que le glucagon élève effectivement l'AMPc cellulaire et active la phosphorylaseakinase (fig. 4).

L'utilisation du propionate pour la néoglucogenèse semble peu altérée par le glucagon (Brockman et Greer, 1980 ; Demigné et al., 1986) ; toutefois Warren et al. (1985), Looney et al. (1987) ont récemment mis en évidence une certaine stimulation.

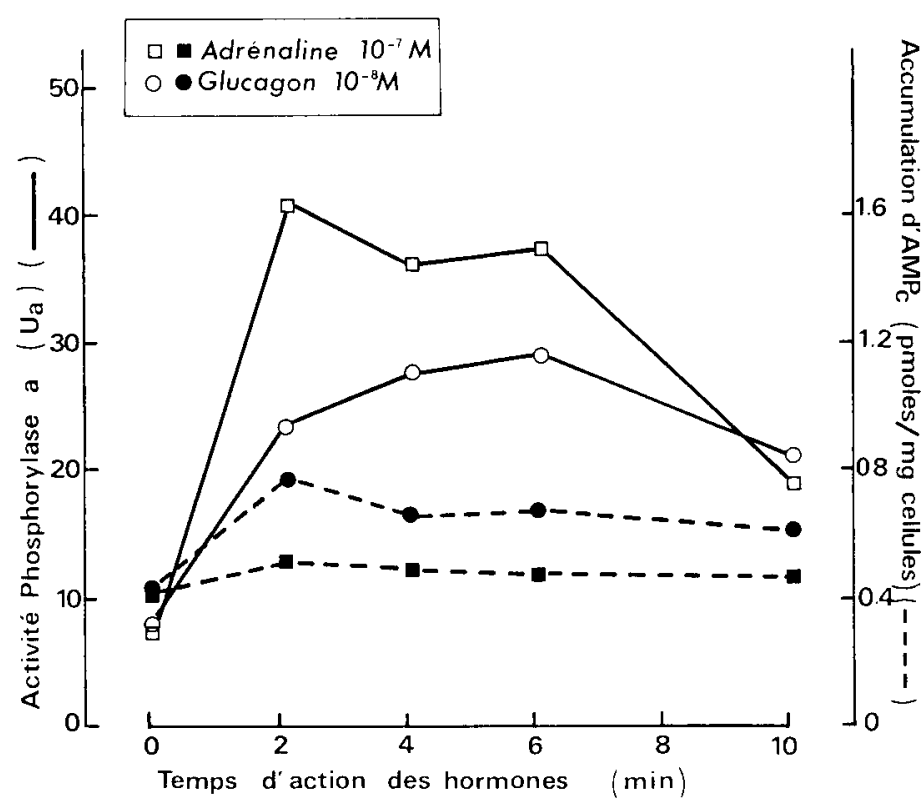

FIG. 4. - Effets du glucagon et de l'adrénaline sur les concentrations en AMPc cellulaires et sur l'activité de la glycogène phosphorylase a sur des hépatocytes isolés de mouton. 
Chez le monogastrique, le glucagon active l'uréogenèse par le biais de l'élévation du transport des acides aminés et par l'activation des enzymes clé. Ches le ruminant, ces points restent à préciser, toutefois, des résultats préliminaires suggèrent des possibilités d'induction du transport, mais de manière beaucoup plus faible que chez le rat. En favorisant l'utilisation des acides aminés pour la synthèse du glucose, moins d'acides aminés sont disponibles pour des tissus extrahépatiques et, à long terme, il existe un effet catabolique.

Dans l'ensemble, le rôle le plus important du glucagon semble être de prévenir l'hypoglycémie postprandiale que pourrait provoquer la sécrétion d'insuline. Enfin, en cas de stress, il participe directement à l'activation de la glycogénolyse hépatique. De plus, il contribue à augmenter la néoglucogenèse lorsque l'insuline et la glycémie sont faibles.

\section{Les catécholamines.}

Le rôle principal des catécholamines est, notamment, de favoriser une fourniture immédiate de glucose et de lipides en cas de stress (hypoglycémie, exercice...). L'adrénaline est un puissant agent hyperglycémiant, et nous avons observé une réponse aux agonistes $\alpha$ et $\beta$. Comme le glucagon, l'adrénaline active la phosphorylase a kinase mais il n'est pas possible de mettre en évidence d'activation d'une étape-clé de la NG par cette hormone. L'adrénaline pourrait élever la NG par le biais des concentrations accrues en lactate et glycérol (lipolyse) qui parviennent au foie. Durant l'administration d'adrénaline, la captation hépatique de lactate augmente 3 ou 4 fois. L'adrénaline déprime aussi la sécrétion d'insuline (Bassett, 1970), et des études sur des moutons pancréatectomisés suggèrent que $50 \%$ des effets hyperglycémiants de l'adrénaline sont attribuables à cet effet pancréatique (Phillips et al., 1969).

\section{Glucocorticoïdes.}

Les glucocorticoïdes jouent un rôle-clé dans le contrôle de la protéolyse, avec un effet antagoniste de l'insuline ; les glucocorticoïdes peuvent d'ailleurs diminuer I'utilisation périphérique du glucose. Les glucocorticoïdes augmentent la glycémie à la fois chez les animaux nourris (Reilly et Black, 1973) et à jeun. En fait, ces hormones jouent un rôle dans la mobilisation des réserves corporelles en favorisant à la fois la protéolyse périphérique et l'uréogenèse hépatique. Elles agissent à ce niveau en synergie avec le glucagon.

\section{Les orientations métaboliques en fonction des états physiologiques.}

II existe des effets très marqués des régimes alimentaires selon les états physiologiques et seuls certains profils endocriniens permettent de stimuler pleinement les fonctions physiologiques impliquées dans les productions animales. Par exemple, c'est en début de lactation que les possibilités de la lipolyse s'expriment au maximum, en relation avec l'élévation du rapport hormone de croissance/insuline et de la sensibilité accrue du tissu adipeux aux catécholamines. 


\section{La croissance.}

La croissance dépend en partie du niveau de la sécrétion d'insuline et donc de la fourniture d'acide propionique, de glucose et d'acides aminés. Les facteurs nutritionnels jouent aussi un rôle déterminant dans la sécrétion hépatique des facteurs de croissance (IGF-1) sous l'influence de l'hormone de croissance. A cet égard, les rations les plus efficaces sont incontestablement celles qui apportent le maximum d'énergie et de protéines digestibles dans l'intestin grêle (PDI).

Les concentrations d'insuline plasmatique sont positivement corrélées à l'ingestion d'énergie. Elles sont plus élevées à l'état nourri lorsque la production de glucose est maximale (Bergman et al., 1970). Paradoxalement, l'extraction du lactate par le foie est faible lorsque la NG est élevée. Il existerait une synergie entre propionate et insuline, qui aboutirait à détourner certains substrats potentiellement glucogéniques (lactate, alanine) vers les tissus extrasplanchniques.

L'insuline, qui est la principale hormone antilipolytique (Brockman et Laarveld, 1986), favorise aussi la lipogenèse par l'orientation du métabolisme de l'adipocyte, la captation d'acétate et de glucose (Jarrett et al., 1974). II semble donc exister une synergie étroite entre l'apport de propionate, de glucose, la sécrétion de l'insuline et la croissance chez les ruminants.

Bien que la disponibilité en acides aminés essentiels soit moins déterminante que chez le monogastrique pour obtenir la croissance maximum, la nature de la ration azotée doit être prise en considération. A cause de leur origine microbienne, il n'y a pas, en dehors de la méthionine, de la lysine et de l'histidine une influence nette de la qualité des protéines sur la protéosynthèse, le facteur limitant résidant dans la quantité d'acides aminés parvenant dans l'intestin grêle (Mc Rae et Lobley, 1984). II faut souligner que la protéosynthèse dépend de la disponibilité en acides aminés mais aussi de l'apport d'énergie. Lorsque la quantité d'acides aminés absorbés dépasse les besoins des synthèses protéiques ou de certains composés (nucléosides, porphyrines), l'excès de chaînons carbonés sera catabolisé. La néoglucogenèse et l'uréogenèse hépatique qui en découlent entraînent une consommation d'ATP accrue au niveau du foie. Le catabolisme des acides aminés a donc pour effet d'augmenter la production d'extra-chaleur et, chez les ruminants comme chez les monogastriques, les régimes hyperprotéiques ne favorisent pas la lipogenèse.

\section{Les orientations du métabolisme durant la gestation et la lactation.}

Durant la gestation, les besoins du conceptus en glucose sont très élevés, et le développement des fœtus est largement tributaire de l'apport de glucose, particulièrement chez les petits ruminants. Comme pour l'animal en croissance, un apport pléthorique de composés glucoformateurs pourra provoquer une lipogenèse excessive avec des risques de mise bas difficile ou de stéatose hépatique en début de lactation (Rémésy et al., 1986). Une restriction énergétique sévère ne provoque pas d'acétonémie malgré la mobilisation des acides gras chez la femelle. Par contre le manque de précurseurs pour la néoglucogenèse se traduit par une forte hypoglycémie-acétonémie lorsque le drainage du glucose par le conceptus ou la glande mammaire est élevé. Pour obtenir une cétogenèse élevée il faut donc 
une carence en composés glucoformateurs très importante qu'il est difficile de reproduire en l'absence de gestation ou de lactation. Les problèmes posés par la lipomobilisation ont été abordés dans une revue récente (Chilliard, 1987).

L'apport optimum en substrats glucoformateurs, en glucose ou en acides aminés (voire en acides gras) est particulièrement difficile à trouver durant la lactation en particulier à cause des différences de potentiel laitier. II existe un effet très net des protéines durant le début de la lactation. Chez ces ruminants en déficit énergétique, il est possible de mettre en évidence l'efficacité d'un apport supplémentaire d'acides aminés (Mc Rae et Lobley, 1984). En effet, un apport modéré de caséine dans la caillette ou de protéines protégées des dégradations microbiennes permet de stimuler la production laitière (Journet et al., 1983). La baisse parallèle des acides aminés non indispensables est en accord avec une stimulation de la néoglucogenèse hépatique. En fait, cet apport supplémentaire en acides aminés semble créer un état favorable à la lactation, ce qui augmente les exportations énergétiques de l'animal et provoque une lipomobilisation accrue lorsque l'ingestion d'énergie est insuffisante. On obtient un effet sensiblement identique par l'utilisation de I'hormone de croissance (Chilliard, 1988). Réciproquement l'administration d'hormone de croissance semble accroître le besoin d'acides aminés, en augmentant la production laitière.

Durant la lactation (comme dans les autres états physiologiques) on peut favoriser la lipogenèse corporelle par un apport excessif de propionate et de glucose, mais ceci est difficile à réaliser tout au moins pendant le premier tiers de la lactation. En fait, afin de ne pas provoquer l'effondrement des matières grasses du lait (par manque de précurseurs à 2 ou 4 atomes de carbones ou par manque d'acides gras longs) on recherche plutôt des fermentations ruminales produisant suffisamment d'acétate et de butyrate. II faut souligner que l'accroissement considérable des quantités ingérées n'entraîne pas pour autant une élévation de l'insulinémie qui reste plutôt basse durant la lactation. L'élévation de l'hormone de croissance aboutit à dériver massivement les nutriments vers la synthèse des constituants du lait. Alors que l'insuline facilite la captation du glucose par le muscle (Jarrett et al., 1974 ; Hay et al., 1984) et le tissu adipeux (Vernon et Finley, 1985), elle ne modifierait pas la captation du glucose par la glande mammaire (Laarveld et al., 1981) ou l'utérus au cours de la gestation (Hay et al., 1983). La faible insulinémie du début de lactation favorise la dérivation du glucose vers la mamelle, en déprimant son utilisation par les autres tissus. Durant la période péripartum, l'anabolisme maternel n'a lieu que si l'absorption de nutriments excède les exigences du conceptus et/ou de la glande mammaire.

Une situation similaire se rencontre avec I'utilisation des acides aminés. Leur captation par le muscle est augmentée par l'insuline (Ahmed et al., 1983), alors que l'effet de l'insuline sur la captation d'acides aminés par la glande mammaire est très faible (Laarveld et al., 1981).

Par le biais de ses effets sur la libération des AGL, l'insuline affecte la cétogenèse. Une réduction de la lipolyse diminue la disponibilité des AGL pour le foie, donc la cétogenèse. Par ailleurs, l'insuline peut supprimer la cétogenèse indépendamment de tout effet sur la concentration en AGL (Brockman et Laarveld, 1986). Pour que la cétogenèse soit élevée, l'insulinémie doit être basse. L'insuline affecte 
la cétogenèse par un autre mécanisme, en favorisant l'utilisation des corps cétoniques (ainsi que de l'acétate) par les tissus extrahépatiques (Jarrett et al., 1974).

La réponse du tissu adipeux à l'insuline s'altère durant la gestation et la lactation. En fin de gestation et en début de lactation, l'insuline est incapable de modifier la lipolyse basale ou après stimulation par des catécholamines (Metz et Van den Bergh, 1977). Dans ces conditions, la lipolyse est très élevée. Les mécanismes de la résistance à l'insuline durant la période autour de la parturition sont mal connus. Cependant, cette résistance est à mettre en relation avec la fourniture d'acétate et d'AGL en vue de la production lactée. Des insulinémies élevées, lorsque les tissus ne sont pas résistants, pourraient déprimer les concentrations d'acétate et d'acides gras dans le sang et par là même diminuer la disponibilité de ces substrats pour les synthèses mammaires.

Chez les animaux en lactation, particulièrement en cas d'hypoglycémiecétose, la réponse de la sécrétion de l'insuline au glucose et au propionate est diminuée (Hove et Halse, 1978 ; Lomax et al., 1979 ; Baird et al., 1980). L'augmentation postprandiale de l'insuline est également réduite (Hove et Halse, 1978) et l'extraction hépatique du lactate est plus élevée que chez les animaux taris. Ainsi, au cours de la lactation, des vitesses maximales de NG peuvent être atteintes et de plus faibles quantités de substrats sont captées par les muscles et le tissu adipeux. La production de lait est ainsi favorisée aux dépens de la croissance corporelle.

En conclusion, il est nécessaire de tenir compte, en plus des apports d'énergie ou d'azote, des produits terminaux de la digestion et de prendre en considération, dans chaque état physiologique, les fonctions que l'on veut favoriser.

3èmes Journées sur la Nutrition et l'Alimentation des Herbivores, I.N.R.A., Paris, 26 et 27 mars 1987.

\section{Références}

AHMED B.M., BERGEN W. G., AMES V. F., 1983. Effect of nutritional state and insulin on hind-limb amino acid metabolism in steers. J. Nutr., 113, 1529-1543.

BAIRD G. D., LOMAX M. A., SYMONDS H. W., SHAW S. R., 1980. Net hepatic uptake and splanchnic metabolism of lactate, pyruvate and propionate in dairy cows in vivo in relation to lactation and nutrient supply. Biochem. J., 186, 45-57.

BASSETT J. M., 1970. Metabolic effects of catecholamines in sheep. Austr. J. biol. Sci., 23, 903-914.

BAUMAN D. E., CURRIE W. B., 1980. Partioning of nutrients during pregnancy and lactation : a review of mechanisms involving homeostasis and homeorhesis. J. Dairy Sci., 63, 1514 1529.

BECK U., EMMANUEL B., GIESECKE D., 1984. The ketogenic effect of glucose in rumen eptithelium of ovine (Ovis aries) and bovine (Bos taurus). Comp. Biochem. Physiol. B., 77, 517 521.

BERGMAN E. N., KATZ M. L., KAUFMAN C. F., 1970. Quantitative aspects of hepatic and portal glucose metabolism and turnover in sheep. Am. J. Physiol, 219, 785-793.

BERGMAN E. N., BROCKMAN R. P., KAUFMAN C. F., 1974. Glucose metabolism in ruminants : comparison of whole body turnover with production by gut, liver and kidney. Fed. Proc., 33 , 1849-1854. 
BRINDLE N. P. J., ZAMMIT V. A., POGSON C. I., 1985. Regulation of carnitine palmitoyl transferase activity by malonyl-CoA in mitochondria from sheep liver, a tissue with a low capacity for fatty acid synthesis. Biochem. J., 232, 177-182.

BROCKMAN R. P., 1985. Role of insulin in regulating hepatic gluconeogenesis in sheep. Can. J. Physiol. Pharmacol., 63, 1460-1464.

BROCKMAN R. P., BERGMAN E. N., 1975. Effect of glucagon on plasma alanine and glutamine metabolism and hepatic gluconeogenesis in sheep. Am. J. Physiol., 228, 1627-1633.

BROCKMAN R. P., GREER C., 1980. Effects of somatostatin and glucagon on the utilization of $\left[2-{ }^{14} \mathrm{C}\right]$ propionate in glucose production in vivo in sheep. Austr. J. Biol. Sci., 33, 457-464.

BROCKMAN R. P., LAARVELD B., 1986. Hormonal regulation of metabolism in ruminants : a review. Livest. Prod. Sci., 14, 313-334.

CHAIYABUTR N., FAULKNER A., PEAKER M., 1983. Effects of exogenous glucose on glucose metabolism in the lactating goat in vivo. Br. J. Nutr., 49, 159-165.

CHILLIARD Y., 1987. Variations quantitatives et métabolisme des lipides dans les tissus adipeux et le foie au cours du cycle gestation-lactation. $2^{\circ}$ partie : chez la brebis et la vache. Reprod. Nutr. Dévelop., 27, 327-398.

CHILLIARD Y., 1988. Rôle et mécanismes d'action de la somatotropine dans l'orientation métabolique du ruminant en lactation. Reprod. Nutr. Dévelop., 28, 39-59.

CLARK M. G., FILSELL O. H., JARRETT I. G., 1976. Gluconeogenesis in isolated intact lamb liver cells. Effects of glucagon and butyrate. Biochem. J., 156, 671-680.

COUDÉ F. X., SWEETMAN L., NYHAN W. L., 1979. Inhibition by propionyl-coenzyme A of N-acetylglutamate synthetase in rat liver mitochondria. J. clin. Invest., 64, 1544-1551.

DEMIGNE C., YACOUB C., REMESSY C., FAFOURNOUX P., 1986. Propionate and butyrate metabolism in rat or sheep hepatocytes. Biochim. Biophys. Acta, 875, 535-542.

DIRKSEN G., LIEBICH H. G., BROSI G., HAGEMEISTER H., MEYER E., 1984. Morphologie des Pansenschleimhaut und Fettsaureresorption beim Rind -bedeutende Faktoren für Gesundheit und Leistung. Zentralb/. Veterinärmed., 31 (A), 414-430.

ELLIOTT J. M., 1980. Propionate metabolism and vitamin B12, 485-503. In RUCKEBUSH Y., THIVEND P., Digestive physiology and metabolism in ruminants, MTP, Lancaster.

FELL B. F., CAMPBELL R. M., MACKIE W. S., WEEKES T. E. C., 1972. Changes associated with pregnancy and lactation in some extra-reproductive organs in the ewe. J. agric. Sci. Cambridge, 79, 397-407

GILL M., BEEVER D. E., 1982. The effect of protein supplementation on digestion and glucose metabolism in young cattle fed on silage. Br. J. Nutr., 48, 37-47.

GRIZARD J., BALAGE M., MANIN M., 1986. Contrôle hormonal du métabolisme hépatique chez le ruminant. Reprod. Nutr. Dévelop., 26, 245-257.

GRIZARD J., ATTAIX D., CHAMPREDON C., BONIN D., ARNAL M., 1988. Oriantations et coordination hormonale du métabolisme protéique chez les ruminants. Reprod. Nutr. Dévelop., 28, 19-37.

HAY W. W., SPARKS J. W., WILKENING R. B., BATTAGLIA F. C., MESCHIA G., 1983. Partition of maternal glucose production between conceptus and maternal tissues in sheep. Am. $J$. Physiol., 245, E347-E350.

HAY W. W., SPARKS J. N., GILBERT M., BATTAGLIA F. G., MESCHIA G., 1984. Effect of insulin on glucose uptake by the maternal hindlimb and uterus, and by the fetus in conscious pregnant sheep. J. Endocrinol., 100, 119-124.

HEITMANN R. N., BERGMAN E. N., 1980. Integration of amino acid metabolism in sheep : effects of fasing and acidosis. Am. J. Physiol., 239, E248-E254.

HEITMANN R. N., DAWES D. J., SENSENIG S. C., 1987. Hepatic ketogenesis and peripheral ketone body utilization in the ruminant. J. Nutr., 117, 1174-1180.

HOVE K., HALSE K., 1978. Absence of feeding induced variations in plasma insulin in hypoglycaemic-ketonaemic cows. Acta vet. scand., 19, 215-228.

HUNTINGTON G. B., PRIOR R. L., BRITTON R. A., 1981. Glucose and lactate absorption and metabolic interrelationships in steers changed from low to high concentrate diets. $J$. Nutr., 111, 1164-1172.

HUNTINGTON G. B., REYNOLDS C. K., 1987. Oxygen consumption and metabolite flux of bovine portal-drained viscera and liver. J. Nutr., 117, 1167-1173. 
JARRETT I. G., FILSELL O. H., BALLARD F. J., 1974. Metabolic and endocrine interrelationships in normal and diabetic sheep. Horm. Metab. Res., 4, 111-116.

JOURNET M., FAVERDIN P., RÉMOND B., VÉRITÉ R., 1983. Niveau et qualité des apports azotés en début de lactation. Bull. tech. C.R.Z.V. Theix, 51, 7-17.

JUDSON G. D., LENG R. A., 1973. The control of gluconeogenesis in sheep: effects of propionate, casein and butyrate infusions. Br. J. Nutr., 29, 175-195.

KATZ J., McGARRY J. D., 1984. The glucose paradox. Is glucose a substrate for liver metabolism ? J. clin. Invest., 74, 1901-1909.

LAARVELD B., CHRISTENSEN D. A., BROCKMAN R. P., 1981. The effect of insulin on net metabolism of glucose and amino acids by the bovine mammary gland. Endocrinology, 108. 2217-2221.

LENG R. A. WEST C. E., 1969. Contribution of acetate, butyrate, palmitate, stearate and oleate to ketone body synthesis in sheep. Res. vet. Sci., 10, 57-63.

LINDSAY D. B., 1982. Relationships between amino acid catabolism and protein anabolism in the ruminant. Fed. Proc., 41, 2550-2554.

LOMAX M. A., BAIRD G. D., MALLINSON C. B., SYMONDS H. W., 1979 . Differences between lactating and non-lactating dairy cows in concentration and secretion rate of insulin. Biochem. J., 180, 281-286.

LOONEY M. C., BALDWIN R. L., CALVERT C. C., 1987. Gluconeogenesis in isolated lamb hepatocytes, J. anim. Sci., 64, 283-294.

LOWE D. M., TUBBS P. K., 1985. Succinylation and inactivation of 3-hydroxy-3-methyl glutarylCoA synthase by succinyl-CoA and its possible relevance to the controle of ketogenesis. Biochem. J., 232, 37-42.

McRAE J. C., LOBLEY G. E., 1984. Interactions between energy and protein, 367-385. In MILLIGAN L. A., GROVUM W. L., DOSSON A., Control of digestion and metabolism in ruminants. Englewood, N.Y.

MARTIN R. J., WILSON L. L., LOWAN R. L., SINK J. D., 1973. Effects of fasting and diet on enzyme profiles in ovine liver and adipose tissue. $J$. anim. Sci., 36, 101-106.

MAUGER J. P., CLARET M., 1986. Mobilization of intracellular calcium by glucagon and cyclic AMP analogues in isolated rat hepatocytes. FEBS Lett., 195, 106-110.

METZ H. M., VAN DEN BERGH S. G., 1977. Regulation of fat metabolism in adipose tissue of dairy cows in the period around parturition. Nether. J. agric. Sci, 25, 198-211.

NAYLOR J. M., KRONFELD D. S., FREEMAN D. E., RICHARDSON D., 1984. Hepatic and extrahepatic lactate metabolism in sheep : effects of lactate loading and $\mathrm{pH}$. Am. J. Physiol., 247, E747-E755.

PHILLIPS R. W., HOUSE W. A., MILLER R. A., MOTT J. L., SOOBY D. L., 1969. Fatty acid, epinephrine and glucagon hyperglycaemia in normal and depancreatectomized sheep. Am. J. Physiol., 217, 1265-1268.

REILLY P. E. B., BLACK A. L., 1973. Early effects of cortisol on glucose and alanine metabolism in adrenalectomized sheep. Am. J. Physiol., 225, 689-695.

RÉMÉSY C., DEMIGNE C., AUFRERE A., 1978. Interorganal relationship of glucose, lactic acid and amino acids in rats fed on high-carbohydrate or high-protein diets. Biochem. J., 170, 321-329.

RÉMÉSY C., CHILLIARD Y., RAYSSIGUIER Y., MAZUR A., DEMIGNÉ C., 1986. Le métabolisme hépatique des glucides et des lipides chez le ruminant : principales interactions durant la gestation et la lactation. Reprod. Nutr. Dévelop., 26, 205-226.

RICKS C. A., COOK R. M., 1978. Volatile fatty acid activation in the young ruminant. Fed. Proc., 37, 411.

SAKATA T., YAJIMA T., 1984. Influence of short-chain fatty acids on the epithelial cell division of digestive tract. Quart J. expt. Physiol., 69, 639-648.

SARTIN J. L., CUMMINS K. A., KEMPPAINEN R. J., MARPLE D. N., RAHE C. H., WILLIAMS J. C., 1985. Glucagon, insulin, and growth hormone responses to glucose infusion in lactating dairy cows. Am. J. Physiol., 248, E108-E114.

SHAW L., ENGEL P. C., 1985 . The suicide inactivation of ox liver short-chain acyl-CoA dehydrogenase by propionyi-CoA. Biochem. J., 230, 723-731.

SMITH V. E., BALDWIN P. L., 1974. Effects of breed, pregnancy, and lactation on weight organs and tissues in dairy cattle. J. Dairy Sci., 57, 1055-1060. 
STANGASSINGER M., GIESECKE D., 1984. Splanchnic metabolism of glucose and related energy substrates, 347-366. In MILLIGAN L. A., GROVUM W. L., DOSSON A. Control of digestion and metabolism in ruminants. Englewood, N.Y.

VAN DER WALT J. G., BAIRD G. D., BERGMAN E. N., 1983. Tissue glucose and lactate metabolism and interconversions in pregnant and lactating sheep. Br. J. Nutr., 50, 267-280.

VERNON R. G., FINLEY E., 1985. Regulation of lipolysis during pregnancy and lactation in sheep. Biochem. J., 230, 651-656.

WARREN G., MTCHELL G. E., BOLING J. A., TUCKER R. E., SCHELLING G. T., DE GREGoRIO R. M., 1985. Glucagon influence on gluconeogenesis and oxidation of propionic acid and threonine by perfused ovine liver. J. Dairy Sci., 68, 2886-2896.

WAHLE K. W. J., LIVESEY C. T., SCAIFE J. R., 1981. Effect of dietary monensin on aspects of propionate metabolism and lipogenesis in sheep, 131-134. In GIESECKE D., DIRKSEN G., STANGASSINGER M. Metabolic disorders in farm animals. Fotodruck Frank OHG, Münich.

WIEGHART M., SLEPETIS R., ELLIOT J. M., SMITH D. F., 1986. Glucose absorption and hepatic gluconeogenesis in dairy cows fed diets varying in forage content. J. Nutr., 116, 839850.

WILTROUT D. W., SATTER L. D., 1972. Contribution of propionate to glucose synthesis in the lactating and non lactating cow. J. Dairy Sci., 55, 307-317.

YACOUB C., RÉMÉSY C., DEMIGNÉ C., 1988. Particularités de l'utilisation des substrats glucoformateurs par les hépatocytes de mouton. Reprod. Nutr. Dévelop., 28, 191-192. 